\title{
Stable Switching of Resistive Random Access Memory on the Nanotip Array Electrodes
}

\author{
Kun-Tong Tsai ${ }^{1,2}$, Chih-Hsiang Ho ${ }^{3}$, Wen-Yuan Chang ${ }^{1}$, Jr-Jian Ke ${ }^{1}$, Elif Selin Mungan ${ }^{3}$, Yuh- \\ Lin $\mathrm{Wang}^{2}$, and Jr-Hau $\mathrm{He}^{1, *}$ \\ ${ }^{1}$ Computer, Electrical and Mathematical Sciences and Engineering (CEMSE) Division, King Abdullah University of \\ Science \& Technology (KAUST), Thuwal 23955-6900, Saudi Arabia \\ ${ }^{2}$ Institute of Atomic and Molecular Sciences, Academia Sinica, P.O. Box 23-166, Taipei 10617, Taiwan \\ ${ }^{3}$ Department of Electrical and Computer Engineering, Purdue University, West Lafayette, IN 47907, USA \\ Email:jrhau.he@kaust.edu.sa
}

Introduction: The formation/rupture of conducting filaments (CFs) in resistive random access memory (ReRAM) materials tune the electrical conductivities non-volatilely and are largely affected by its material composition [1], internal configurations [2] and external environments [3,4]. Therefore, controlling repetitive formation/rupture of CF as well as the spatial uniformity of formed CF are fundamentally important for improving the resistive switching (RS) performance. In this context, we have shown that by adding a field initiator, typically a textured electrode, both performance and switching uniformity of ReRAMs can be improved dramatically [5]. In addition, despite its promising characteristics, the scalable fabrication and structural homogeneity of such nanostructured electrodes are still lacking or unattainable, making miniaturization of ReRAM devices an exceeding challenge. Here, we employ nanostructured electrode (nanotip arrays, extremely uniform) formed spontaneously via a self-organized process to improve the $\mathrm{ZnO}$ ReRAM switching characteristics.

Device Fabrication: Fig. 1(a) illustrates the configuration of fabricated ReRAM. An AFM image of a patterned substrate comprising abundance of $\mathrm{Al}$ nanotips is shown in Fig. 1(b). It was fabricated using anodization of $\mathrm{Al}$ at 25 $\mathrm{V}$ in sulfuric acid to generate self-organized nano-architectures $[6,7]$ and the subsequently chemical etching to remove alumina intact with arrays of periodic Al nanotips (Fig. 2). We then stacked the $\mathrm{ZnO}$ RS layer (100 nm) and Pt top electrode $(100 \mathrm{~nm})$ on the patterned $\mathrm{Al}$ bottom electrode (hereafter referred as patterned sample for the simplicity). The cross-sectional SEM image in Fig. 1(c) clearly shows that the deposited films are conformal to the patterned Al substrate. For the comparison, parallel preparation and measurement of the ReRAM devices fabricated on the polished Al substrate (flat sample) are also characterized (Fig. 1(d)).

Results: Both patterned and flat sample exhibit a typical bipolar switching as shown in Fig. 3. The resistance state translated from high resistance state (HRS) to low resistance state (LRS) denotes a set process at set voltage $\left(\mathrm{V}_{\text {set }}\right)$, while the translation from LRS to HRS denotes the reset process at reset voltage $\left(\mathrm{V}_{\text {reset }}\right)$. Current compliance (C.C.) protects devices from prominent degrade. The flesh devices do not show any bistable switching. An electroforming process is necessary to activate the RS switching at a forming voltage $\left(\mathrm{V}_{\mathrm{f}}\right)$ of all devices (Fig.4(a)). Compared to the flat sample, patterned sample tends to switch at lower $\mathrm{V}_{\text {set }}$ with a smaller set current (Fig. 3). It therefore enlarges the window for the multilevel storage as shown in the inset of Fig. 3. From the statistic analysis of $V_{f}$ in Fig. 4(b), we observe that the patterned sample has a lower $V_{f}$. The patterned samples show an average $V_{f}$ of $4.7 \mathrm{~V}$, whereas the flat samples show a $38 \%$ higher $V_{f}$ of $6.5 \mathrm{~V}$. Such lowering of $V_{f}$ could result from the increase number of defects, larger contact area, and higher electric field. However, since the decreased IRS, which normally comes with the increase number of defects and larger contact area, is not observed (Fig. 4(c)), we can safely conclude that the decreased $V_{f}$ is mainly from the high electric field. It is also confirmed by Sentaurus simulations. In Fig. 5, a concentrated electric field is ambiguously shown at the apex of the nanotip. Such locally boosted electric-field can give the spatial control of the nucleation of CFs, which is otherwise difficult to control at the flat sample with homogenous features. It brings tremendous advantages such as uniform switching characteristics, as shown in narrower cumulative probability distributions of $\mathrm{V}_{\text {set }}$ (Fig. 6). Furthermore, the $60 \%$ lowering of $\mathrm{V}_{\text {set }}$ in patterned sample is closely associated with the modification of the switch transportation under such a higher electric field stress (Fig. 7), which is dominated by a weak space charge limited current (SCLC).

Conclusion: We have demonstrated that $\mathrm{ZnO}$ resistive memory with a nanostructured substrate has great potential in improving ReRAM's RS characteristics. The electric field concentrated on nanotip structures is believed to play a crucial role for lowering $\mathrm{V}_{\mathrm{f}}$ and $\mathrm{V}_{\text {set. }}$. The uniformity of the nanostructures is also important for the optimization of device performance, as well as improving the switching uniformity and reliability. Combining with the fact that fabrication process has low-cost merit with excellent stability and scalability, the nanotip array is highly attractive for cost-effective ReRAM applications and for the device miniaturization.

References:

[2] R. Waser et al., Adv. Mater., vol. 21, p. 2632, (2009).

[4] D.-H. Lien et al., Nanoscale, vol. 7, p. 19874, (2015).

[6] K.-T. Tsai et al., Nano Lett., vol. 14, p. 4971, (2014).
[1] W.-Y. Chang et al., Appl. Phys. Lett., vol. 92, p. 022110, (2008).

[3] J.-J. Ke et al., Appl. Phys. Lett., vol. 99, p. 192106, (2011).

[5] Q. Liu et al., ACS Nano, vol. 4, p. 6162, (2010).

[7] W. Lee et al., Chem. Rev. vol. 114, p. 7487, (2014). 


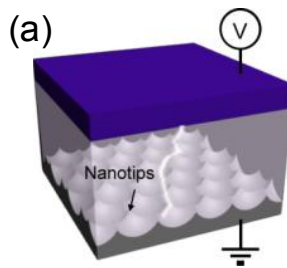

(c)

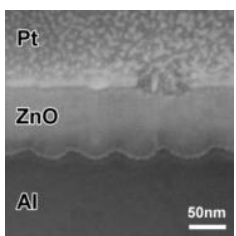

(b)

$12 \mathrm{~nm}(\mathrm{~d})$
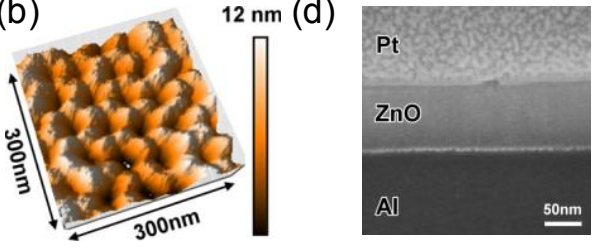

Fig. 1. (a) Configuration of the fabricated ReRAM and schematics of electrical characterization. (b) AFM image of the patterned substrate with scalable self-organized Al nanotips on its surface. Crosssectional SEM image of the (c) patterned sample and (d) flat sample.

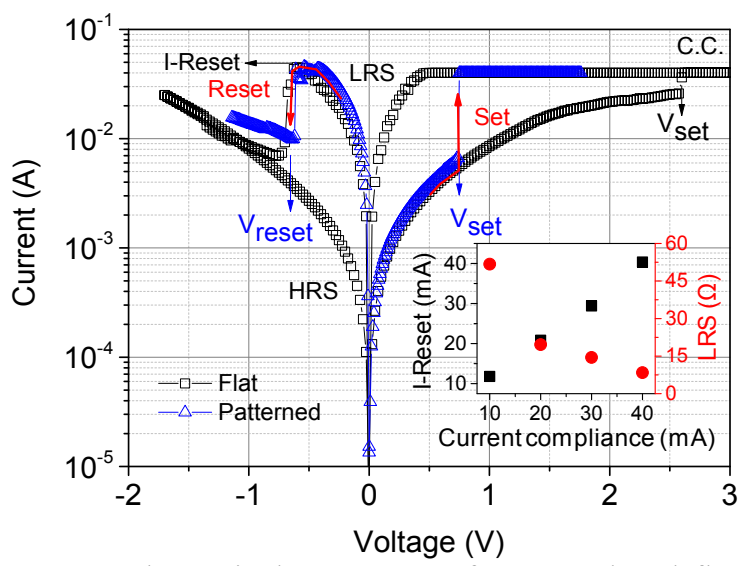

Fig. 3. The typical I-V curve of patterned and flat sample. Inset plot shows the multilevel storage with larger capacity in patterned sample.

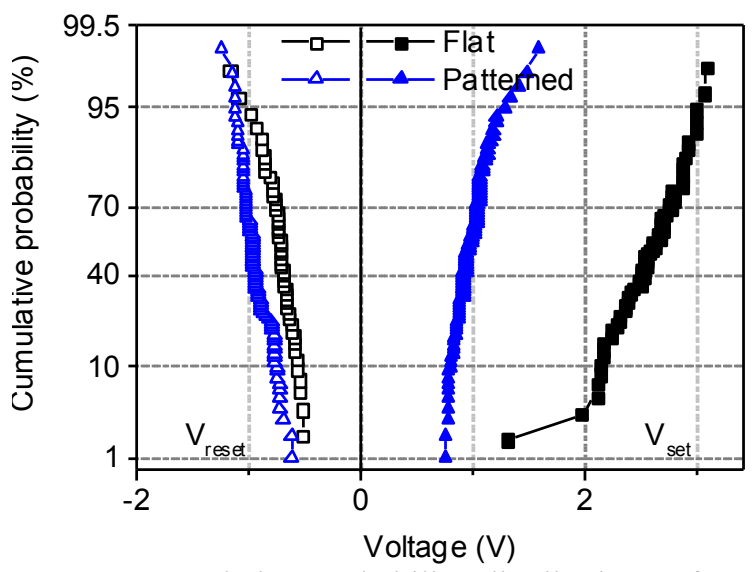

Fig. 6. Cumulative probability distributions of $\mathrm{V}_{\text {set }}$ and $V_{\text {reset }}$ observed on both the flat and patterned devices.

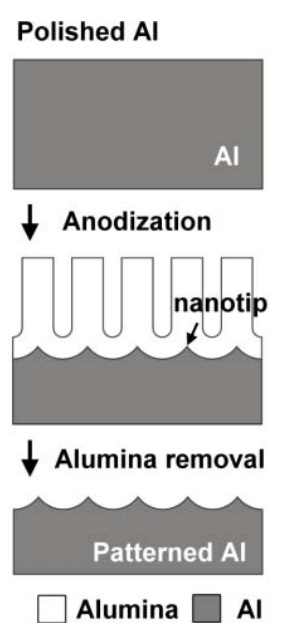

Fig. 2. Fabrication procedure for creating $\mathrm{Al}$ patterned substrate.

(a)
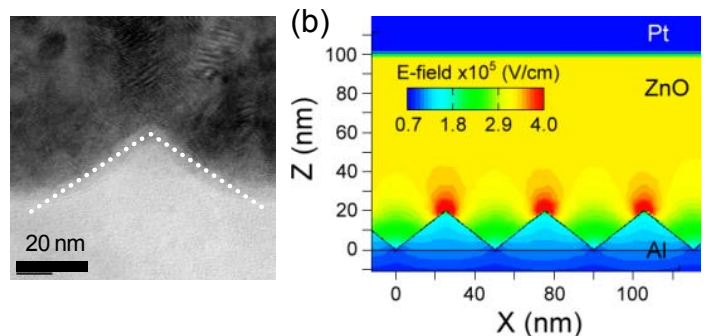

Fig. 5. (a) TEM image of the nanotips architecture. (b) Sentaurus simulation showing the E-field distribution of the patterned sample.

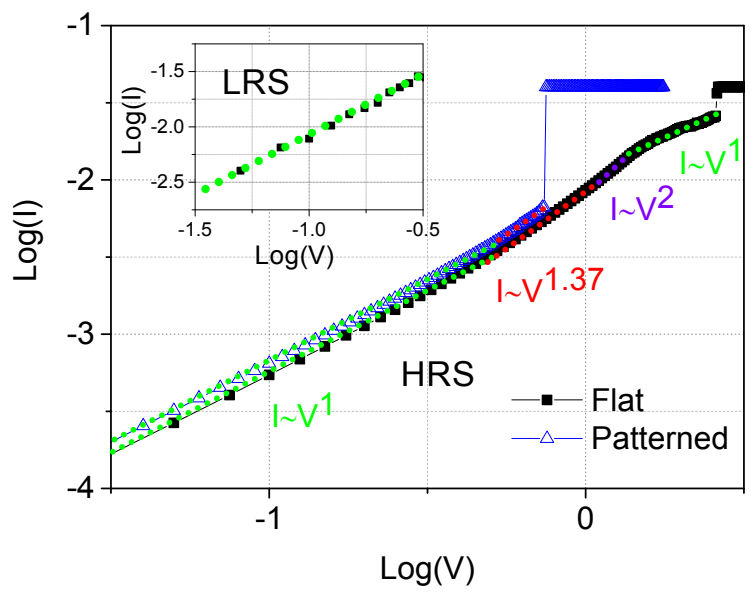

Fig. 7. The log-log plot of the I-V curve showing the transportation mechanism of the switching. HRS at flat sample is dominant by SCLC. Current conduction follows Ohm's law ( $I \sim V^{1}$; slop of 1$)$ in low voltage region, while it corresponds to Child's law $\left(I \sim V^{2}\right.$; slope of 2) in high voltage region. Disappearing of $\mathrm{I} \sim \mathrm{V}^{2}$ at HRS in the patterned sample represents a weak SCLC. LRS for the flat and patterned devices shows a linear transient of the ohmic behavior. 